\title{
Type-4 Phosphodiesterase (PDE4) Blockade Reduces NETosis in Cystic Fibrosis
}

Licia Totani ${ }^{1 \star}$, Concetta Amore ${ }^{1}$, Antonio Piccoli ${ }^{1}$, Giuseppe Dell'Elba ${ }^{1}$, Angelo Di Santo ${ }^{1}$, Roberto Plebani ${ }^{2}$, Romina Pecce ${ }^{2}$, Nicola Martelli ${ }^{1}$, Alice Rossi ${ }^{3}$, Serena Ranucci ${ }^{3}$, Ida De Fino ${ }^{3}$, Paolo Moretti ${ }^{4}$, Alessandra Bragonzi ${ }^{3}$, Mario Romano ${ }^{2 \star}$ and Virgilio Evangelista ${ }^{1 *}$

${ }^{1}$ Laboratory of Vascular Biology and Pharmacology, Fondazione Mario Negri Sud, Santa Maria Imbaro (CH), Mozzagrogna, Italy, ${ }^{2}$ Laboratory of Molecular Medicine, Centre for Advanced Studies and Technology (CAST), Department of Medical Oral and Biotechnological Sciences, G. D'Annunzio University of Chieti-Pescara, Chieti, Italy, Infection and Cystic Fibrosis Unit, Division of Immunology Transplantation and Infectious Diseases, IRCCS San Raffaele Scientific Institute, Milan, Italy, ${ }^{4}$ Cystic Fibrosis Centre, S. Liberatore Hospital, Atri, Italy

\section{OPEN ACCESS}

Edited by: Angelo Sala,

University of Milan, Italy

Reviewed by: Fabrizio Facchinetti, Chiesi Farmaceutici, Italy Pierachille Santus, University of Milan, Italy

*Correspondence: Licia Totani totani58licia@gmail.com Mario Romano mromano@unich.it Virgilio Evangelista evangelista59gino@gmail.com

Specialty section: This article was submitted to Inflammation Pharmacology a section of the journal

Frontiers in Pharmacology

Received: 29 April 2021

Accepted: 26 July 2021

Published: 08 September 2021

Citation:

Totani L, Amore C, Piccoli A, Dell'Elba G, Di Santo A, Plebani R,

Pecce R, Martelli N, Rossi A, Ranucci $S$, De Fino I, Moretti $P$, Bragonzi A, Romano $M$ and Evangelista V (2021) Type-4 Phosphodiesterase (PDE4) Blockade Reduces NETosis in Cystic Fibrosis.

Front. Pharmacol. 12:702677. doi: 10.3389/fphar.2021.702677
Neutrophilic inflammation is a key determinant of cystic fibrosis (CF) lung disease. Neutrophil-derived free DNA, released in the form of extracellular traps (NETs), significantly correlates with impaired lung function in patients with $\mathrm{CF}$, underlying their pathogenetic role in CF lung disease. Thus, specific approaches to control NETosis of neutrophils migrated into the lungs may be clinically relevant in CF. We investigated the efficacy of phosphodiesterase (PDE) type-4 inhibitors, in vitro, on NET release by neutrophils from healthy volunteers and individuals with CF, and in vivo, on NET accumulation and lung inflammation in mice infected with Pseudomonas aeruginosa. PDE4 blockade curbed endotoxin-induced NET production and preserved cellular integrity and apoptosis in neutrophils, from healthy subjects and patients with CF, challenged with endotoxin, in vitro. The pharmacological effects of PDE4 inhibitors were significantly more evident on CF neutrophils. In a mouse model of Pseudomonas aeruginosa chronic infection, aerosol treatment with roflumilast, a selective PDE4 inhibitor, gave a significant reduction in free DNA in the BALF. This was accompanied by reduced citrullination of histone $\mathrm{H} 3$ in neutrophils migrated into the airways. Roflumilast-treated mice showed a significant improvement in weight recovery. Our study provides the first evidence that PDE4 blockade controls NETosis in vitro and in vivo, in CF-relevant models. Since selective PDE4 inhibitors have been recently approved for the treatment of COPD and psoriasis, our present results encourage clinical trials to test the efficacy of this class of drugs in $\mathrm{CF}$.

\section{Keywords: cystic fibrosis, neutrophil, neutrophil extracellular traps, lung damage, PDE4 inhibition}

\section{INTRODUCTION}

Neutrophilic inflammation is the trademark of cystic fibrosis (CF) lung disease (Tirouvanziam, 2006). Excessive and persistent accumulation of neutrophils in the airways, associated with impaired bacterial clearance and tissue damage, is the early event in the life of patients with CF (Davis and Ferkol, 2013; Sly et al., 2013). In CF airways, neutrophils release proteases, mainly elastase that 
induces inflammatory responses, impairs ciliary function in epithelial cells, disables CXCL8-induced bacterial killing, and causes bronchomalacia and bronchiectasis (Hartl et al., 2007; Davis and Ferkol, 2013).

In addition, recruited neutrophils produce the so-called neutrophil extracellular traps (NETs), consisting of a nuclear DNA backbone decorated with granular enzymes that help to capture and kill, extracellularly, invading bacteria (Brinkmann et al., 2004). However, recent observations in preclinical and clinical CF models indicate that the excessive accumulation of NETs in the airways plays a key pathogenetic role in lung disease (Cheng and Palaniyar, 2013). Abundant NETs can be found in the airways of people with $\mathrm{CF}$ and significantly correlate with impaired lung function, suggesting that excessive NETosis, in CF, may act as a double-edged sword between host-defense and autoinflammation (Marcos et al., 2015). Indeed, more recent observations show that Pseudomonas aeruginosa isolated from patients with CF is resistant to the bactericidal activity of NETs (Young et al., 2011). Moreover, excessive NET formation may play a pathogenetic role in vasculitis (Kessenbrock et al., 2009) and provide a scaffold for platelet adhesion and thrombus formation (Fuchs et al., 2010), thus mediating micro- and macrovascular occlusion.

The basic mechanisms of vital NETosis have been recently uncovered. Upon appropriate stimulation of neutrophils, the nuclear envelope disintegrates and allows mixing of chromatin with granular enzymes, such as myeloperoxidase and elastase, which together with type IV peptidyl-arginine deiminase (PAD) promotes chromatin decondensation before extracellular release of a large filament of DNA-enzyme complexes, as NETs (Neeli et al., 2008; Wang et al., 2009; Papayannopoulos et al., 2010; Thiam et al., 2020). Although the discovery of the process of NETosis and of the underlying mechanisms is relatively recent, its pharmacological modulation remains largely unknown. While PAD4 inhibitors, such as GSK484 (Mondal and Thompson, 2019), BMS-P5 (Li et al., 2020), or the more historic Clamidine, are described in the literature, they remain in a nonclinical stage so far. Thus, current therapies for CF lack specific approaches to tackle excessive NETosis.

Type 4 phosphodiesterases (PDE4s), the major isoform of PDEs expressed by myeloid cells, control a variety of inflammatory mechanisms in immune cells. In neutrophils, PDE4s are key mediators of cAMP degradation and, as a downstream effect, of neutrophil adhesion and migration, cytokine and chemokine release, synthesis of lipid mediators, and of reactive oxygen species (Sanz et al., 2005). In several animal models, genetic deficiency of PDE4 reduces neutrophilic inflammation (Jin and Conti, 2002; Ariga et al., 2004; Jin et al., 2005). In agreement with genetic ablation, pharmacological blockade of PDE4 reduces leukocyte recruitment at the site of inflammation (Sanz et al., 2002; Sanz et al., 2007). Moreover, it was reported that PDE4 blockade promotes neutrophil apoptosis, thus driving resolution of inflammation (Sousa et al., 2010). From a mechanistic point of view, we have recently discovered that selective blockade of PDE4 in human neutrophils downregulates Src family kinase activities (SFK), through protein kinase A (PKA)-mediated activation of $\mathrm{COOH}$-terminal Src kinase
(CSK), a major endogenous regulator of SFK (Totani et al., 2014). Through these mechanisms, roflumilast, an oral selective PDE4 inhibitor approved for clinical use in patients with severe chronic obstructive pulmonary disease, prevents the release of NETs from neutrophils adhered on fibrinogen and challenged with bacterial endotoxin (Totani et al., 2016).

Here, we tested the hypothesis that PDE4 inhibitors may control NETosis in CF. The efficacy of PDE4 blockade was explored in vitro, using CF neutrophils, and in vivo, in a mouse model of Pseudomonas aeruginosa chronic lung infection.

\section{MATERIALS AND METHODS}

\section{Chemicals and Reagents}

Selective PDE-4 inhibitors tested in this study were roflumilast$\mathrm{N}$-Oxide (RNO), the active metabolite of roflumilast (Bundschuh et al., 2001), kindly provided by Nycomed-Takeda (Konstanz, Germany), roflumilast for in vivo experiments by Aurogene (Rome, Italy), and rolipram by Calbiochem (Milan, Italy).

\section{Human Volunteers}

Healthy donors were recruited among staff members of the Fondazione Mario Negri Sud. All donors signed an informed consent form. Individuals with CF (demographic characteristics are reported in Table 1) were recruited at the Cystic Fibrosis Center of Atri Hospital (TE, Italy) on an outpatient basis. Patients and their parents were informed about the rationale and objectives of the study and asked to sign the relative form. The experimental protocol was communicated to the Ethic Committee of the Institution, according to the national guidelines (G.U. n. 76 del 31-03-2008). Inclusion criteria: no antibiotics or steroids for at least 2 weeks before blood collection. Blood $(15 \mathrm{ml})$ was collected in the occasion of a scheduled routine control. Pulmonary function was evaluated after a suspension of at least $12 \mathrm{~h}$ of bronchodilator or leukotriene receptor antagonist administration.

\section{Neutrophil Isolation and Incubation}

Neutrophils were isolated from the citrated whole blood by standard procedures routinely used in our laboratory (Evangelista et al., 2007). In order to mimic CFTR dysfunction, normal neutrophils were treated with CFTRinh$172(10 \mu \mathrm{Mol} / \mathrm{L})$ for $15 \mathrm{~min}$ before experimental use. For adhesion, DMSO or CFTRinh-172-treated neutrophils (4 $\mathrm{x}$ $10^{6} / \mathrm{ml}$ ), resuspended in HEPES-Tyrode buffer ( $\mathrm{pH} 7.4$ ) containing $129 \mathrm{mmol} / \mathrm{L} \quad \mathrm{NaCl}, \quad 9.9 \mathrm{mmol} / \mathrm{L} \quad \mathrm{NaHCO}_{3}$, $2.8 \mathrm{mmol} / \mathrm{L} \quad \mathrm{KCI}, \quad 0.8 \mathrm{mmol} / \mathrm{L} \quad \mathrm{KH}_{2} \mathrm{PO}_{4}, \quad 0.8 \mathrm{mmol} / \mathrm{L} \mathrm{MgCl}-$ $6 \mathrm{H}_{2} \mathrm{O}, 5.6 \mathrm{mmol} / \mathrm{L}$ Dextrose, $10 \mathrm{mmol} / \mathrm{L}$ HEPES, and $1 \mathrm{mmol} /$ $\mathrm{L} \mathrm{CaCl}_{2}$ were seeded on fibrinogen-coated $(200 \mu \mathrm{g} / \mathrm{ml} ; 200 \mu \mathrm{l} /$ well for $24 \mathrm{~h}$ at $\left.4^{\circ} \mathrm{C}\right) 12$-well plates and allowed to adhere at $37^{\circ} \mathrm{C}$, $5 \% \mathrm{CO}_{2}$, in the absence or presence of bacterial endotoxin from Escherichia coli, serotype 055:B5 (Sigma-Aldrich, Milan, Italy) $(10 \mu \mathrm{g} / \mathrm{ml})$ for $18 \mathrm{~h}$ (Totani et al., 2016). In initial experiments, in order to set up the model, neutrophils were also stimulated by the classical chemoattractants fMLP (1 $\mu$ Moles/L) and C5a (1 $\mu$ Moles/L) or phorbol 12-myristate 13-acetate (PMA) (100 
TABLE 1 | Demographic characteristics of subjects with CF recruited for the study.

\begin{tabular}{lcll}
\hline Age (Genotype) & $\mathbf{F E V}_{\mathbf{1}} \mathbf{\%}$ & Chronic infection & Discontinuous infection \\
\hline 27 years (G85E/R75X) & 46,2 & S.A. & P.A. \\
20 years (F508del/1303K) & 38,1 & S.A., P.A. & P.A. \\
17 years (UNK) & 46,2 & S.A., P.A., P.M., A.F. & A.F. \\
44 years (F508del/G542X) & 25,3 & B.C. & P.A. \\
40 years (F508del/G542X) & 41,7 & S.A., P.A. & \\
17 years (F508del/F508del) & 92,0 & B.C. & S.A. \\
22 years (F508del/F508del) & 111,6 &
\end{tabular}

SA, Staphylococcus aureus; PA, Pseudomonas aeruginosa; BC, Burkholderia cenocepacia; PM, Proteus mirabilis; and AF, Aspergillus fumigatus.

nMoles/L). When indicated, roflumilast-N-oxide (RNO) or a vehicle (DMSO) was added to cells 2 min before seeding.

\section{Neutrophil Extracellular Trap Measurements}

DNA-NETs formed by neutrophils adherent to fibrinogen-coated surfaces were visualized by confocal microscopy after staining DNA with DRAQ-5 and FITC-conjugated anti-myeloperoxidase antibodies (Abcam, Cambridge, United Kingdom) (Totani et al., 2016). Extracellular DNA was quantified by a Quant-iT ${ }^{\mathrm{TM}} \mathrm{dsDNA}$ high-sensitivity assay kit (Molecular Probes by Life Technologies, Oregon, United States) as previously described (Totani et al., 2016). Briefly, $2.5 \mu \mathrm{l}$ of endonuclease (nuclease micrococcal, from Staphylococcus aureus $50 \mathrm{U} / \mathrm{ml}$ ) (Sigma-Aldrich, Milan, Italy) was added to cell samples $(500 \mu \mathrm{l})$ and incubated at $37^{\circ} \mathrm{C}$ for $10 \mathrm{~min}$, followed by addition of $5 \mu \mathrm{l}$ of EDTA $(0.5 \mathrm{M})$ to stop the reaction. Samples were centrifuged at $10.000 \mathrm{x} g$ in an Eppendorf centrifuge for $3 \mathrm{~min}$, and supernatants stored at $-20^{\circ} \mathrm{C}$ until DNA quantification.

\section{Citrullinated Histone-3 Detection}

Pellets of BAL neutrophils $\left(1 \times 10^{5}\right)$ were lysed with RIPA buffer. Pools of BAL supernatants or of cell lysates from all animals per each treatment group were analyzed by Western blotting using an anti-histone H3 (citrulline R2+R8+R17) (ab5103Abcam, Cambridge, Mass., United States).

\section{Immunoblotting}

For Western blot analysis, the reaction was stopped by adding an equal volume of $2 \mathrm{x}$ reducing Laemmli's lysis buffer, containing $2 \mathrm{mmol} / \mathrm{L}$ sodium orthovanadate, $5 \mathrm{mmol} / \mathrm{L}$ EGTA, $5 \mathrm{mmol} / \mathrm{L}$ EDTA, $10 \mathrm{mmol} / \mathrm{L}$ sodium pyrophosphate, $10 \mathrm{mmol} / \mathrm{L}$ iodoacetic acid, $1 \mathrm{mmol} / \mathrm{L}$ phenylmethylsulphonyl fluoride, $10 \mathrm{mmol} / \mathrm{L}$ sodium fluoride, $10 \mu \mathrm{g} / \mathrm{ml}$ leupeptin and aprotinin, and $1 \mathrm{mg} /$ $\mathrm{ml}$ trypsin/chymotrypsin inhibitors, to the cell suspension. Samples were boiled for $10 \mathrm{~min}$ and centrifuged for $10 \mathrm{~min}$ at $10,000 \mathrm{~g}$. Aliquots of $100 \mu \mathrm{l}$, corresponding to $0.2 \times 10^{6}$ neutrophil total lysates (the supernatant and cells), were loaded into $10 \%$ gradient sodium dodecyl sulfate-polyacrylamide gel. Proteins were transferred onto nitrocellulose sheets, and nonspecific sites were blocked using $1 \%$ bovine serum albumin (BSA) in Tris-buffered saline overnight at room temperature on a horizontal shaker. The presence of citrullinated histone- 3 was analyzed by immunoblotting with a rabbit, polyclonal, anti-histone $\mathrm{H} 3$ (citrulline R2+R8+R17) (ab5103Abcam, Cambridge, Mass., United States) $(1: 1,000 ; 1 \mathrm{~h}$ at room temperature) specific antibody, followed by incubation with an anti-rabbit ECLconjugated secondary antibody $(1: 5,000 ; 1 \mathrm{~h}$ at room temperature). The ECL reagent (Perkin/Elmer, Inc) (MA/ United States) was used for the detection of luminescence, by UVITEK.

\section{Flow Cytometry}

Neutrophils were analyzed by flow cytometry to quantify the percentage of neutrophils: 1) remaining morphologically intact, 2) showing markers of apoptosis, and 3) containing myeloperoxidase, after $18 \mathrm{~h}$ of adhesion to fibrinogen-coated surfaces. To this end, neutrophils were detached from fibrinogen by a brief exposure to EDTA/EGTA (both $5 \mathrm{mMol} /$ L). Neutrophil apoptosis was quantified by a FITC-conjugated annexin-V/propidium iodide (P.I.) kit according to the manufacturer's instruction (BD-Biosciences, United States). The intracellular myeloperoxidase content was measured by the "MPO-FITC" Kit according to the manufacturer's instruction (Beckman Coulter, Milan, Italy).

\section{Animal Studies}

Animal studies adhered strictly to the Italian Ministry of Health guidelines for the use and care of experimental animals (protocol \#549 and 733). Research with P. aeruginosa RP73 isolate from CF individuals has been approved by the Ethics Commission of Hannover Medical School, Germany. The patient and parents gave informed consent before the sample collection. Approval for storing biological materials was obtained by the Ethics Commission of Hannover Medical School, Germany. C57Bl/ 6NCrlBR male mice (8-10 weeks of age) from Charles River Laboratories were challenged with $1 \times 10^{6} \mathrm{CFU}$ of MDR-RP73 embedded in agar beads for chronic infection by intratracheal administration, as previously described (Bragonzi, 2010; Paroni et al., 2013; Facchini et al., 2014).

First, mice were treated by gavage with roflumilast $(5 \mathrm{mg} / \mathrm{kg})$ or a vehicle (4.4\%DMSO in saline) daily, starting $2 \mathrm{~h}$ before infection. Health and body weight were monitored daily. Mice were killed 5 days after infection, $2 \mathrm{~h}$ after the last treatment. The lungs were excised and analyzed for bacterial loads, by measuring the colony forming units (CFUs). The bronchoalveolar lavage fluid (BALF) was analyzed for the total and differential cell count, amount of free DNA, and cytokine content. Free DNA was measured by a Quant-iT ${ }^{\mathrm{TM}}$ dsDNA high-sensitivity assay kit as 


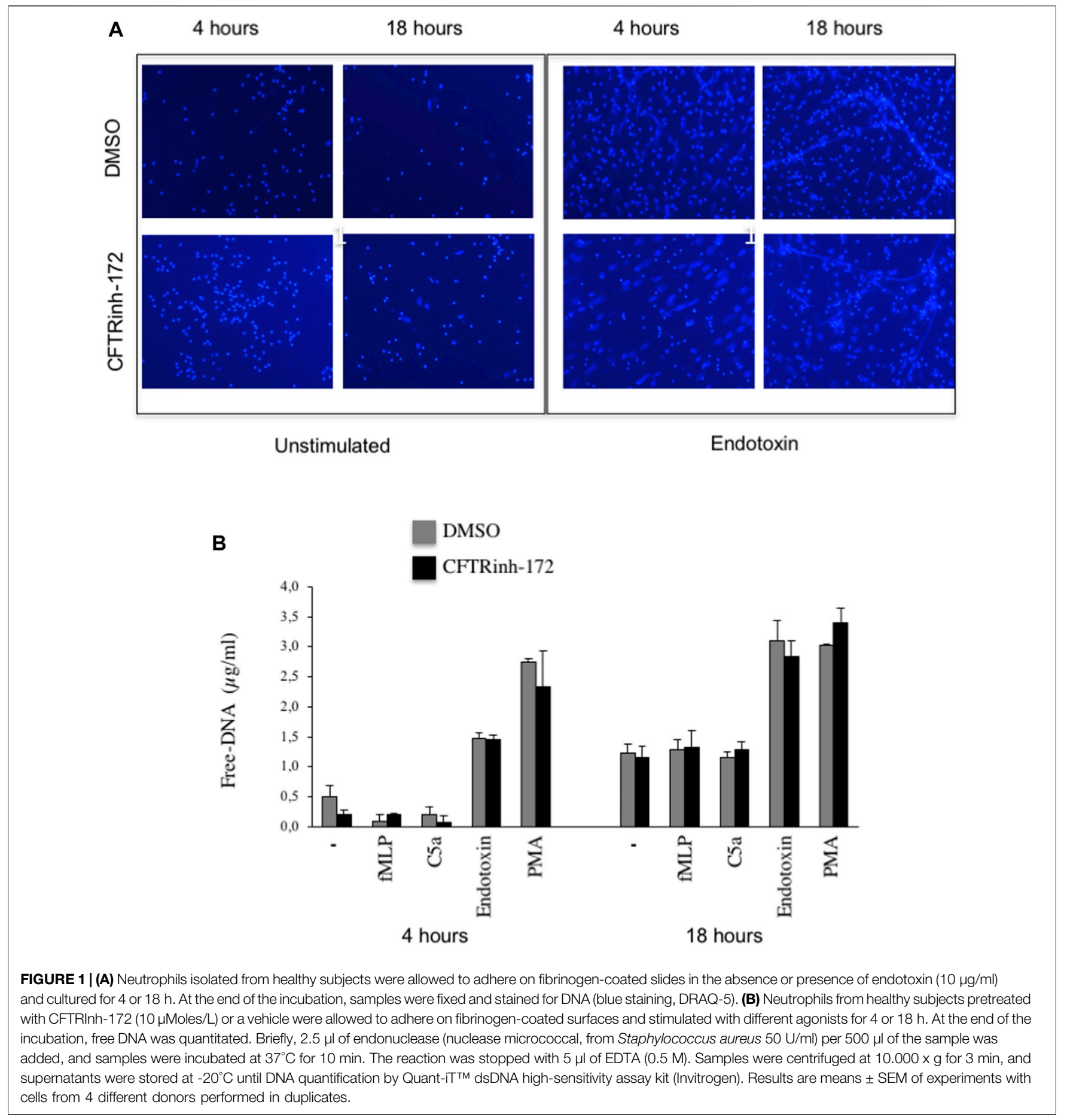

followed for in vitro experiments. Cytokines were measured using a competitive ELISA method or a Luminex multi-analyte assay (ProcartaPlex, Thermo Fisher Scientific, Monza, Italy).

Next, mice were treated per aerosol with roflumilast $(5 \mathrm{mg} / \mathrm{kg})$ or a vehicle $(4,4 \%$ DMSO in saline) using Penn Century as previously described (Cutone et al., 2019). The drug or vehicle was administered once a day, starting from $4 \mathrm{~h}$ after infection. Each group of treatment was divided into two: one group of animals was killed $28 \mathrm{~h}$ after infection ( $2 \mathrm{~h}$ after treatment) to analyze the effect of treatments on the acute phase of the infection, whereas the other group was killed 5 days after infection (i.e., $2 \mathrm{~h}$ after the last treatment) to analyze the effect of treatments in chronic infection.

Body weight was determined, and aerosol administration was carried out once a day under anesthesia (5\% isoflurane-oxygen, running at $4 \mathrm{l} / \mathrm{min}$ ) according to established procedures. At the 
end of the experiment, the BALF was collected and analyzed for cells, free DNA, and cytokine content. A fraction of the BALF containing a fixed number of neutrophils $\left(\begin{array}{lll}1 & \mathrm{x} & 10^{5}\end{array}\right)$ was centrifuged, and the pelleted cells were immediately frozen and stored for Western blot analysis of citrullinated histone H3. The lungs were excised and homogenized, and CFU counting was performed as reported (Bragonzi, 2010; Paroni et al., 2013; Facchini et al., 2014; Cutone et al., 2019).

\section{Statistical Analysis}

Data from in vitro experiments were reported as the mean and standard deviation. The paired $t$-test was used to analyze the differences between treated and untreated cell samples. An analysis-of-variance (ANOVA), accounting for correlation within matched pairs, was used to explore concentration-response relationships. $p$-values $<0.05$ were considered statistically significant. Data from in vivo experiments are reported as box plots: the horizontal line in the middle of the box marks the median of values; the edges of each box mark the 25th and 75th percentiles; and vertical lines, extending up and down from each box, represent largest and smallest values, respectively, that are not outliers (values greater than 1.5 times the length of the box were considered outliers and excluded from the analysis). Paired $t$-test was used analyze the differences between treated and untreated groups. $p$-values $<0.05$ were considered statistically significant.

\section{RESULTS}

\section{PDE4 Blockade Prevents Neutrophil Extracellular Trap Release}

To analyze NET release in vitro, we stimulated neutrophils, adhered on fibrinogen-coated surfaces, with bacterial endotoxin from Escherichia coli at $37^{\circ} \mathrm{C}, 5 \% \mathrm{CO}_{2}$. NETs were visualized by confocal microscopy after DNA staining. After $4 \mathrm{~h}$ of stimulation with endotoxin, most of the neutrophils showed decondensation of their nuclei, and DNA appeared widespread into the cytoplasm and outside the cell (Figure 1A). Few extracellular DNA filaments were visible at this early time point. After $18 \mathrm{~h}$, macroscopic NET structures (Figure 1A) were diffused among cells. In order to explore the potential role of CFTR in NETosis, we evaluated, in parallel, NET formation in neutrophils that were untreated or exposed to CFTRinh-172, a selective CFTR inhibitor. As shown in Figure 1A, NET formation was not apparently influenced by CFTR inhibition. For a more quantitative analysis, extracellular free DNA was measured in supernatants. Released DNA was barely detectable after $4 \mathrm{~h}$ in unstimulated cells, and it was not significantly enhanced by the classical chemoattractants fMLP and C5a. A substantial increment was instead observed in the presence of endotoxin or PMA (Figure 1B). A similar pattern was observed after $18 \mathrm{~h}$ of incubation (Figure 1B). Free-DNA quantitation confirmed that CFTR inhibition did not significantly influence NET release (Figure 1B).

Next, we examined the impact of PDE4 blockade on neutrophil morphology and DNA release by confocal

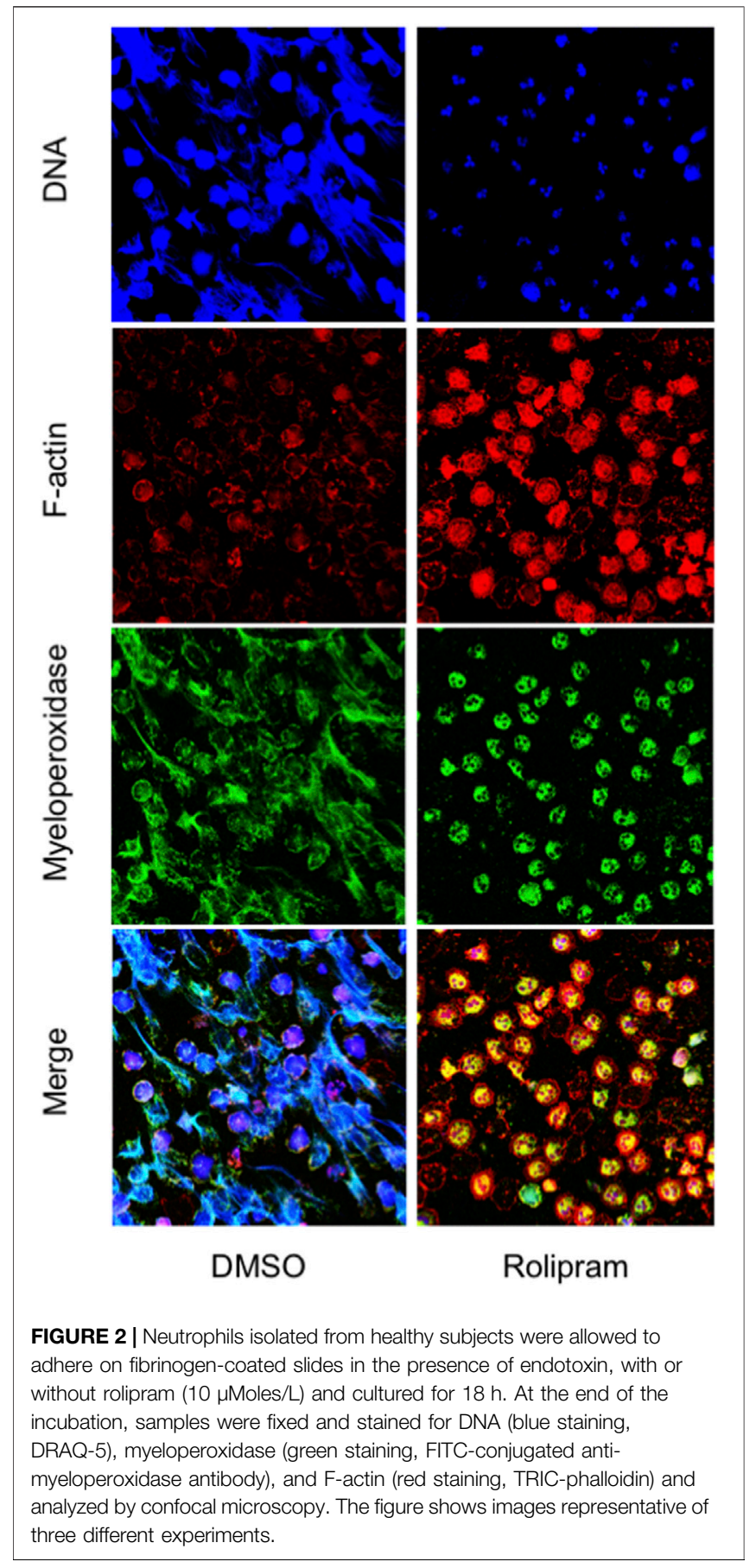

microscopy and free-DNA quantitation. NET structures were visualized by DNA and myeloperoxidase staining with DRAQ-5 and an FITC-conjugated anti-myeloperoxidase antibody, respectively. After $18 \mathrm{~h}$ of incubation, DNA filaments decorated with myeloperoxidase were diffused among cells. Notably, rolipram $(10 \mu \mathrm{M})$ preserved morphological integrity, nuclear DNA, and cytoplasmic myeloperoxidase localization (Figure 2). Consistently, RNO (the active metabolite of roflumilast) concentration-dependently $(0.1-1 \mathrm{Mol} / \mathrm{L})$ reduced DNA release by neutrophils from healthy volunteers as this effect 
A
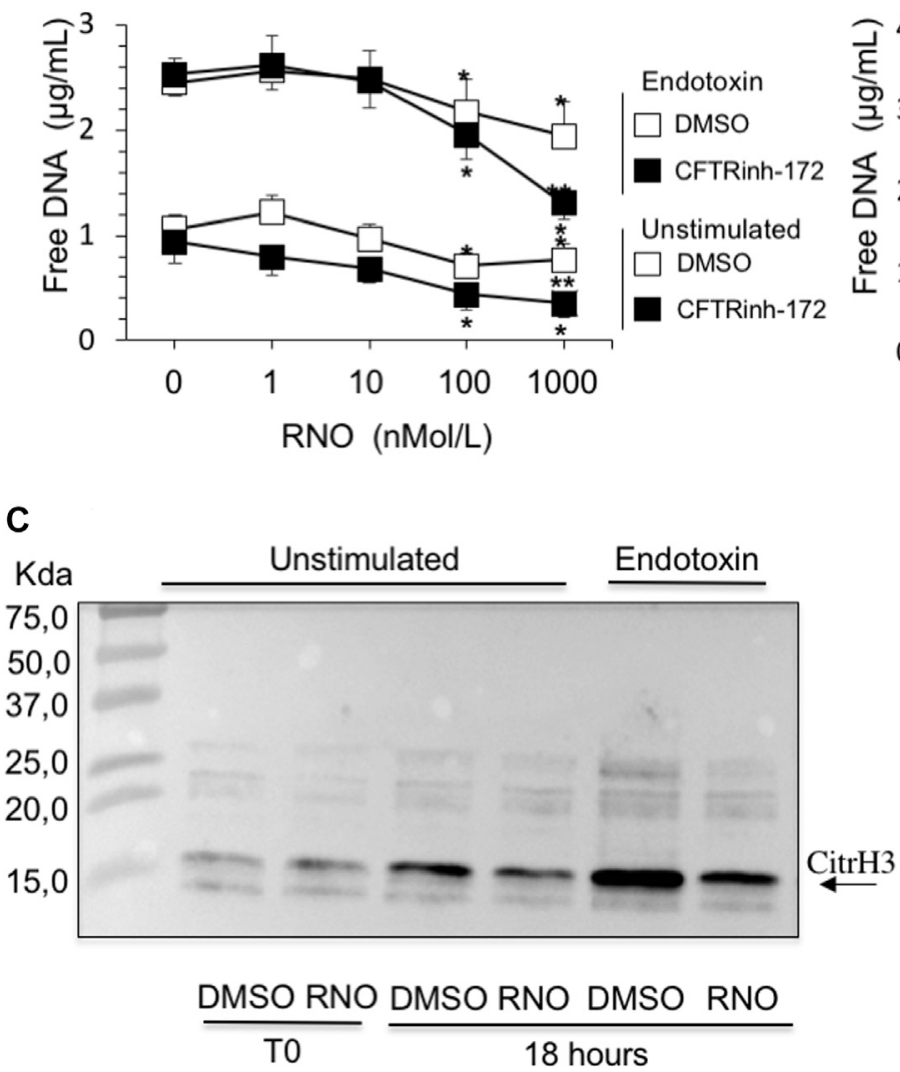

B

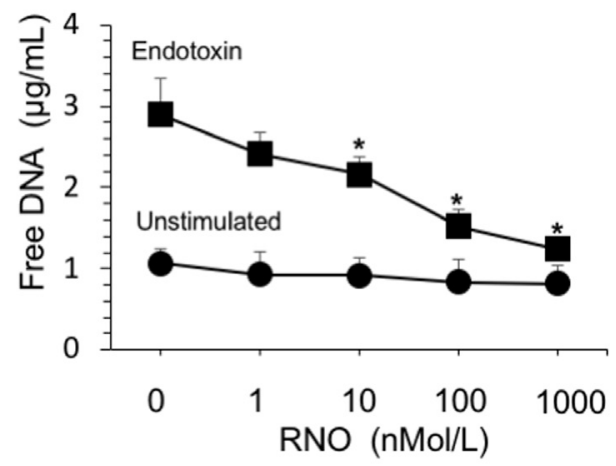

D Unstimulated
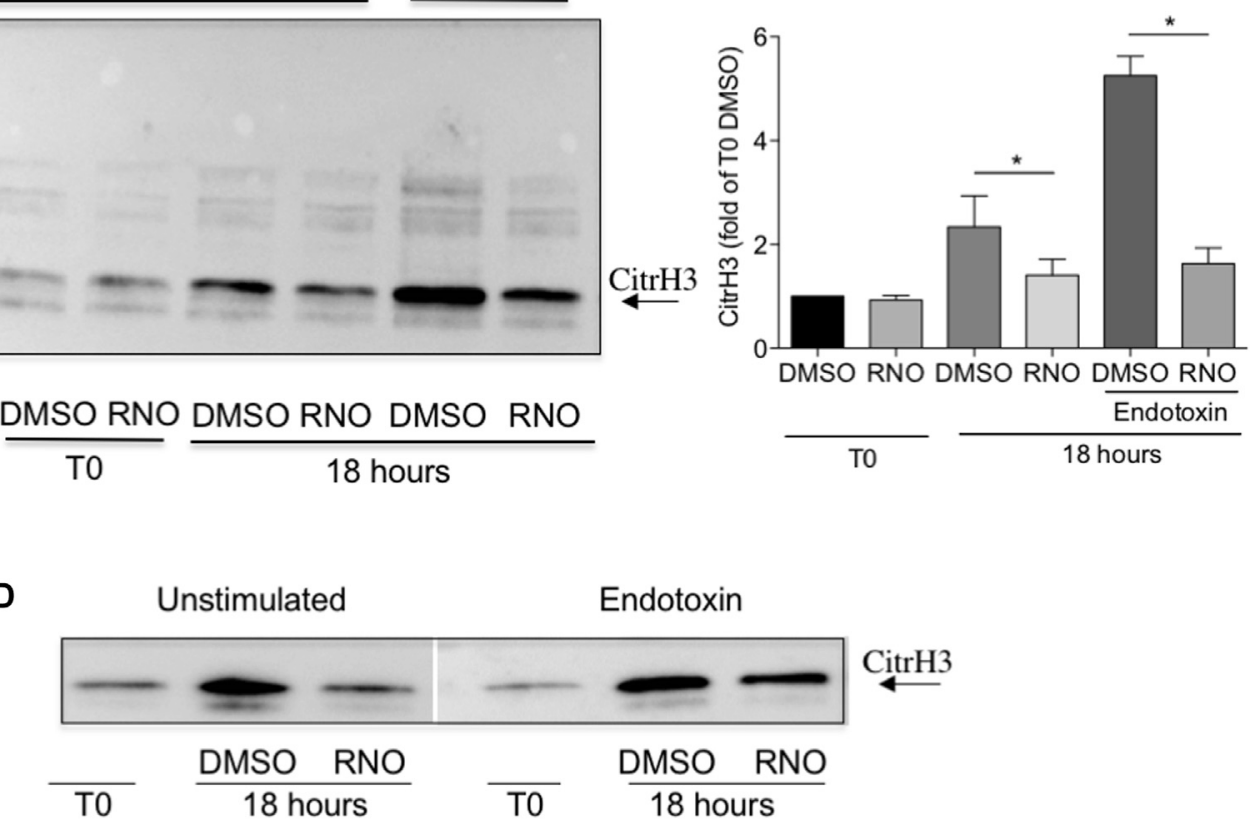

FIGURE 3 | (A) Neutrophils from healthy subjects, pretreated for 2 min with increasing concentrations of RNO (0-1,000 nMoles/L), were exposed to endotoxin and allowed to adhere on fibrinogen-coated surfaces for $18 \mathrm{~h}$ in the presence or in the absence of CFTRinh-172 (10 $\mu$ Moles/L). Unstimulated neutrophils, pretreated with increasing concentrations of RNO (0-1,000 nMoles/L), were incubated in parallel. At the end of the incubation, free DNA was quantitated. Results are mean \pm SEM of experiments performed with cells from 9-11 different donors in duplicates. ${ }^{*} p<0.05$ (ANOVA, Dunnett test), RNO-treated vs untreated samples; ${ }^{* *} p<0.05$ (Student's t-test), CFTRinh-172-treated vs untreated samples. (B) Neutrophils from individuals with CF, pretreated with increasing concentrations of RNO (0-1,000 nMoles/L), were incubated with endotoxin and allowed to adhere on fibrinogen-coated surfaces for $18 \mathrm{~h}$. Unstimulated neutrophils, pretreated with increasing concentrations of RNO (0-1,000 nMoles/L), were incubated in parallel. Results are mean \pm SEM of experiments performed with cells from seven different patients with CF (see Table 1 for patients' characteristics). ${ }^{*} p<0.05$ (ANOVA, Dunnett test) vs untreated samples. The presence of citrullinated histone H3 in neutrophils from three healthy donors (C) or two people with CF (D) was analyzed after $18 \mathrm{~h}$ of incubation. Samples were then subjected to Western blot analysis using a monoclonal antibody which specifically recognizes citrullinated histone $\mathrm{H} 3$. Representative Western blots are shown. The right image in panel $\mathrm{C}$ reports a densitometric analysis from $\mathrm{n}=3$. ${ }^{\star} p<0.05$ (Student's t-test).

was more evident in the presence of CFTRinh-172 (Figure 3A). Similar measurements were carried out with neutrophils isolated from seven patients with CF. As shown in Figure 3B, RNO concentration-dependently reduced free-DNA release.

NET formation requires the activation of peptidylarginine deiminase 4 (PAD4) that converts arginine to citrulline on nuclear histones and promotes chromatin decondensation. Thus, histone $\mathrm{H} 3$ citrullination may be considered a specific
NETosis marker (Wang et al., 2009; Papayannopoulos et al., 2010; Thiam et al., 2020). To unequivocally confirm that reduction of free DNA was a consequence of inhibition of NETosis, we assessed the presence of citrullinated histone $\mathrm{H} 3$ in cell lysates from neutrophils isolated from healthy volunteers and people with CF by Western blot analysis. Figure 3 shows that RNO reduced citrullination of histone $\mathrm{H} 3$, demonstrating that PDE4 blockade controls biochemical events necessary for NET 


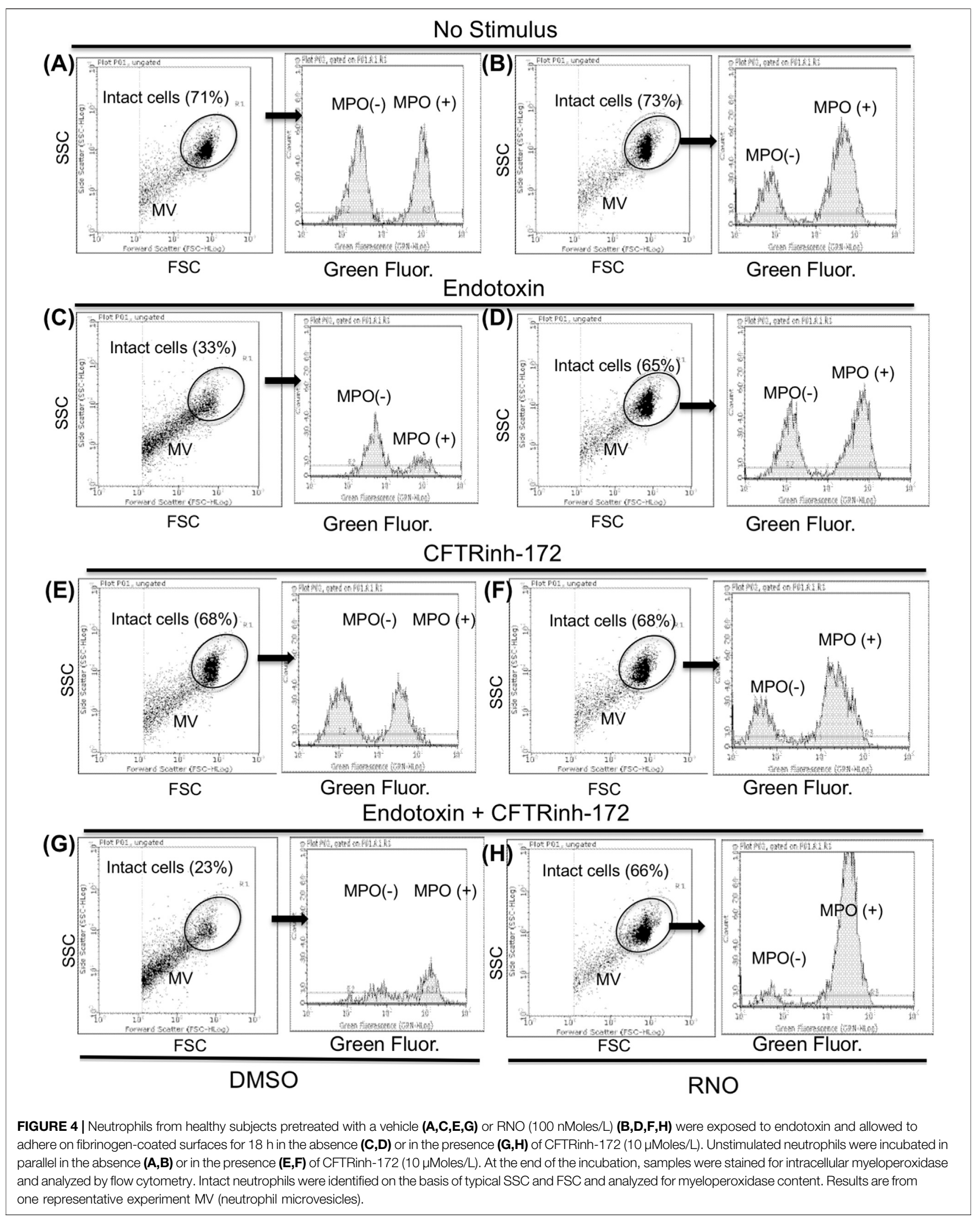




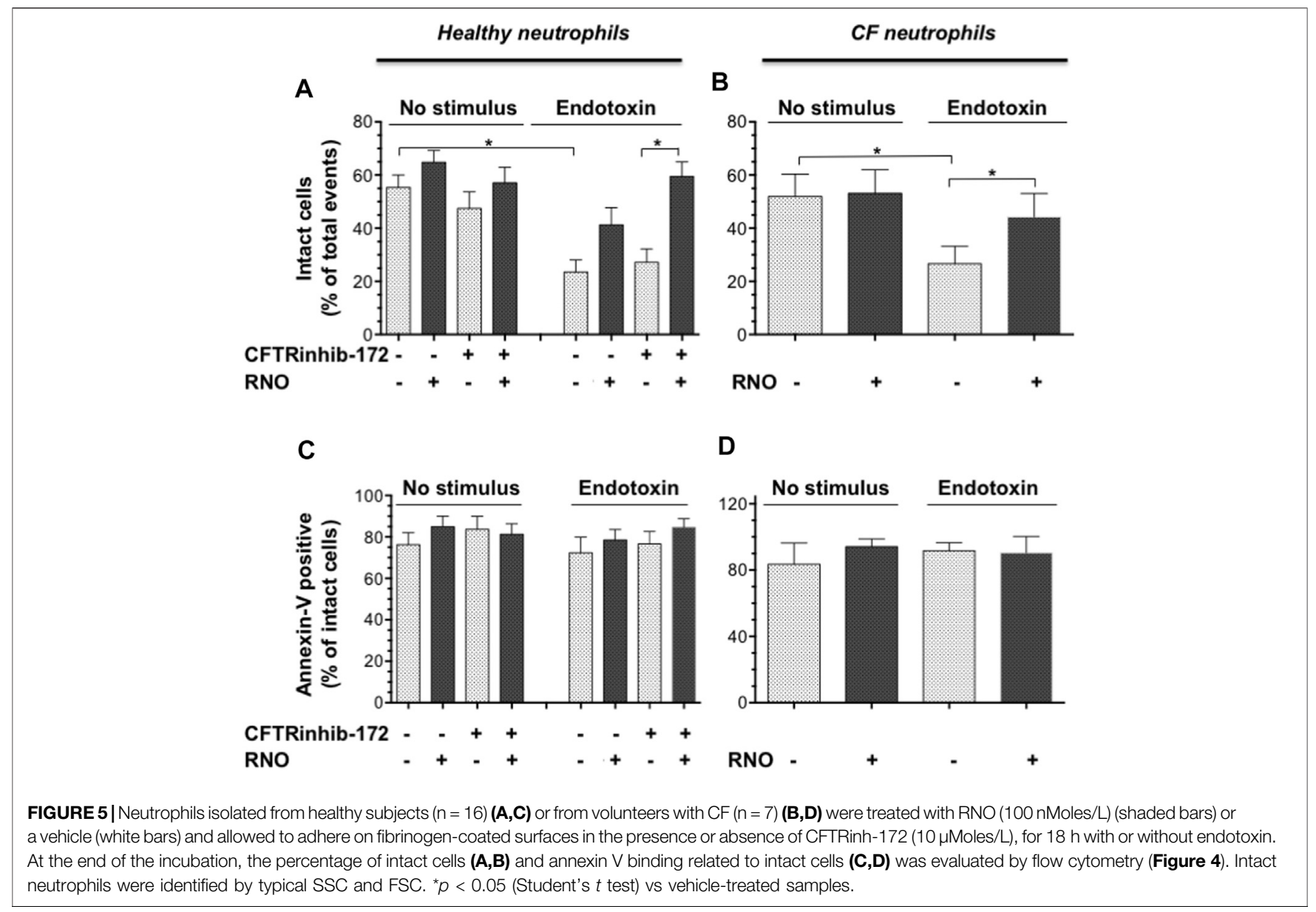

formation in healthy (Figure 3C) and CF (Figure 3D) neutrophils.

\section{PDE4 Blockade Preserves Neutrophil Integrity and Apoptosis}

NETosis is accompanied by microvesiculation and fragmentation of netting neutrophils (Thiam et al., 2020). Images shown in Figure 2 are suggestive of protective effects by the PDE4 inhibitor on neutrophil fragmentation. To obtain a more quantitative readout of this effect, we used flow cytometry. To this end, after $18 \mathrm{~h}$ of adhesion, neutrophils from normal volunteers were detached from fibrinogen-coated surfaces by a brief exposure to $5 \mathrm{mMol} / \mathrm{L}$ EGTA/EDTA, permeabilized, and stained with a FITC-conjugated anti-myeloperoxidase antibody. Figure 4 reports a flow cytometric analysis showing that, compared to unstimulated samples (A), endotoxin stimulation (C) significantly decreased the percentage of intact neutrophils. Moreover, in the absence of endotoxin, $50 \%$ of the intact neutrophil population contained large amounts of intracellular myeloperoxidase (A), indicating the presence of nondegranulated cells. In contrast, in endotoxin-treated samples, the few neutrophils remaining intact appeared completely degranulated (C). No significant changes were detected in the presence of CFTRinh-172 (Figures 4E and G, without or with endotoxin, respectively). PDE4 inhibition by RNO (100 nMoles/L) significantly increased the percentage of intact neutrophils as well as of non-degranulated cells in all experimental settings (Figures 4B,D,F, and $\mathbf{H}$, and Figure 5A). These effects were quantitated in neutrophils from seven donors with $\mathrm{CF}$ and 16 healthy volunteers. We consistently observed that RNO (100 nMoles/L) increased the percentage of intact neutrophils in endotoxin-stimulated samples (Figures 5A,B). Since neutrophil apoptosis is key for the proper development of the resolution program of the inflammatory response and neutrophils from patients with CF manifest delayed apoptosis (McKeon et al., 2008; Moriceau et al., 2010; Gray et al., 2018), we asked whether preservation of neutrophil integrity by PDE4 inhibition had an impact on neutrophil apoptosis. For this purpose, neutrophils were stained with annexin $\mathrm{V}$ and P.I. to assess the percentage of early/late apoptotic or necrotic cells. Results reported in Supplementary Tables S1,S2 clearly show that the majority of intact neutrophils, both healthy and $\mathrm{CF}$, were annexin $\mathrm{V}$ positive/P.I negative; a lower number showed annexin $\mathrm{V}$ positive/P.I. positive. In contrast, the fraction of viable (annexin $\mathrm{V}$ negative/P.I. negative) or necrotic cells (annexin $\mathrm{V}$ negative/P.I. positive) was negligible. Figures $5 \mathbf{C}$ and $\mathbf{D}$ summarize that 
TABLE 2 | Effect of oral administration of roflumilast on CFUs in the lungs and inflammatory markers in the BALF.

\begin{tabular}{lcc}
\hline & Vehicle & Roflumilast \\
\hline Total cells $\left(10^{6} / \mathrm{ml}\right)$ & $2,49 \pm 2,1$ & $1,57 \pm 1,2(p=0,04)$ \\
Neutrophils $\left(10^{6} / \mathrm{ml}\right)$ & $1,84 \pm 1,0$ & $1,04 \pm 1,14(p=0,05)$ \\
Macrophages $\left(10^{6} / \mathrm{ml}\right)$ & $0,52 \pm 0,2$ & $0,48 \pm 0,4(p=0,41)$ \\
Interleukin-1 $\beta(\mathrm{pg} / \mathrm{ml})$ & $114,3 \pm 51,2$ & $80,27 \pm 69,0(p=0,10)$ \\
TNF- $\alpha(\mathrm{pg} / \mathrm{ml})$ & $1506,9 \pm 263,9$ & $1184,4 \pm 392,1(p=0.008)$ \\
CXCL-1 $(\mathrm{KC})(\mathrm{pg} / \mathrm{ml})$ & $19,0 \pm 9,0$ & $13,7 \pm 11,1(p=0,12)$ \\
Free DNA $(\mu / \mathrm{ml})$ & $2,2 \pm 2,3$ & $1,5 \pm 1,4(p=0,19)$ \\
CFU - lung $(\times 1,000)$ & $49,6 \pm 52,9$ & $55,9 \pm 91,7(p=0,47)$ \\
Total proteins $(\mathrm{mg} / \mathrm{ml})$ & $558,7 \pm 208,3$ & $445,8 \pm 119,3(p=0,07)$
\end{tabular}

C57BL/6 male mice (8-10 weeks of age) were infected i.t. with $1 \times 10^{6}$ CFUs of MDR$R P 73$ embedded in agar beads. Mice (11 per group of treatment) were treated by gavage with roflumilast (5 mg/kg/day) or placebo (4,4\% DMSO in saline), with the starting dose administered $2 \mathrm{~h}$ before infection and the last $2 \mathrm{~h}$ before killing. 5 days after infection, mice were sacrificed, the BALF was collected, and mouse lungs were recovered, homogenized, and plated to determine the bacterial load. Counts of total cells, neutrophils, and macrophages, as well as levels of cytokines and total protein, were evaluated in the BALF. Statistical differences between roflumilast- and vehicle-treated animals were analyzed by Student's t-test.

approximately $80 \%$ of endotoxin-stimulated normal ( \pm CFTRinh172) or CF neutrophils, which remained intact after $18 \mathrm{~h}$, displayed an apoptotic profile. Collectively, these results suggest that PDE4 inhibition controlling NETosis and, at the same time, preserving neutrophil apoptosis may be useful to mitigate neutrophilic inflammation in CF. To further support this hypothesis, we conducted preclinical studies in a mouse model of bacterial lung inflammation.

\section{Effect of PDE4 Inhibition on Lung Inflammation and Infection in Mice}

First, the efficacy of oral administration of roflumilast was tested in a murine model of Pseudomonas aeruginosa infection to mimic a chronic lung infection similar to the one typically established in the airways of people with CF. C57Bl/6NCrlBR mice were challenged with $1 \times 10^{6}$ MDR-RP73 embedded in agar beads by intratracheal administration to induce chronic infection. Mice were treated with roflumilast $(5 \mathrm{mg} / \mathrm{kg}$ ) or a vehicle $(4,4 \%$ DMSO in saline) by gavage once a day for 5 days, starting $2 \mathrm{~h}$ before infection. Body weight and health of mice were monitored daily. After 5 days of infection ( $2 \mathrm{~h}$ after the last treatment), mice were killed, and the BALF was collected and analyzed for the total and differential cell count, protein content, as markers of vascular permeability, and interleukin (IL)- $1 \beta$, tumor necrosis factor (TNF)- $\alpha$, and KC, the analogue of IL-8 in the mouse, levels as indices of inflammation. The amount of free DNA in the BALF was analyzed as an indirect measurement of NETs. In addition, since treatments that impair neutrophil activities may potentially reduce immune responses to bacterial infection, CFUs were counted in BAL and in homogenized lung tissue. The effects of oral administration of roflumilast on bacterial loads and inflammatory (cells and cytokines) markers in the BALF are summarized in Table 2. No difference was observed in the lung CFU after 5 days of infection between roflumilast- and vehicle-treated animals. We observed a clear trend toward a reduction in the inflammatory response in the roflumilast-treated group compared to the vehicle-treated group. Statistically significant differences were detected in total cells and in the number of neutrophils, but no differences were observed in the number of macrophages. Roflumilast treatment induced a statistically significant reduction in TNF- $\alpha$, while the reduction in $\mathrm{KC}$ and IL-1 $\beta$ levels was not significant. Free DNA and total protein content also appeared to be reduced in the BALF of treated mice, but the differences did not reach the statistical significance. Based on these results, we reasoned that, compared with the oral route, intratracheal administration could yield higher drug concentration in the airways and more effectively control neutrophil recruitment and activation. Three doses of roflumilast $(0.5,1$, or $5 \mathrm{mg} / \mathrm{kg} /$ day $)$ were tested for toxicity in noninfected animals. No evidence of side effects was recorded for all doses (data not shown). Therefore, we explored the efficacy of intratracheal administration of roflumilast $(5 \mathrm{mg} / \mathrm{kg} /$ day for 5 days) in C57Bl/6NCrlBR mice infected with Pseudomonas aeruginosa MDR-RP73 embedded in agar beads. To exclude possible effects of the drug during the initial phase of bacterial infection, the first treatment was started $4 \mathrm{~h}$ after infection. For each group of treatment, a half number of animals were killed $28 \mathrm{~h}$ after the infection, $2 \mathrm{~h}$ after the second treatment, to analyze the effect of the drug in the acute phase, and the remaining were killed 5 days after infection, $2 \mathrm{~h}$ after the last treatment. At these time points, the BALF and lungs were collected for analyses. We observed that the number of total cells, neutrophils, and macrophages, increased at 5 days with respect to $28 \mathrm{~h}$, in the vehicle-treated group (Figures 6A-C), indicating persistence of cell recruitment. Treatment with roflumilast, which did not modify inflammatory cells in the BALF at $28 \mathrm{~h}$ of infection, significantly reduced the number of total cells and the number of neutrophils at 5 days post infection compared to vehicle-treated animals (Figures 6A,B). On the contrary, the macrophage count was not affected by roflumilast (Figure 6C). Moreover, roflumilast-treated mice showed significantly lower amount of free DNA in the BALF at 5 days, compared with vehicle-treated animals (Figure 7A). To unequivocally confirm that the reduction in free DNA was a consequence of NETosis inhibition, we analyzed the presence of citrullinated histone $\mathrm{H} 3$ in supernatants and lysates from neutrophils recovered in the BALF. To this purpose, pools of supernatants and cell lysates from all BALFs of each group were subjected to the Western blot analysis of citrullinated histone $\mathrm{H} 3$. As shown in Figures 7B and C, citrullinated histone H3, which was undetectable in samples collected after $28 \mathrm{~h}$ from infection, increased both in supernatants and inside the cells in samples collected 5 days after infection. At this time point, BALF supernatants and cells collected from animals treated with roflumilast displayed reduced citrullinated histone $\mathrm{H} 3$ compared to vehicle-treated mice, conclusively demonstrating that PDE4 inhibition controls DNA release in inflamed airways by blocking biochemical events necessary for NET formation. Measurements of body weight, as an index of the general health status of infected animals, indicated a rapid decrease in body weight in both groups of treatment. The group of mice treated with roflumilast recovered weight more rapidly than the vehicletreated group (Figure 8A). The improvement in recovery was statistically significant 5 days after infection (Figure $\mathbf{8 B}$ ). The 


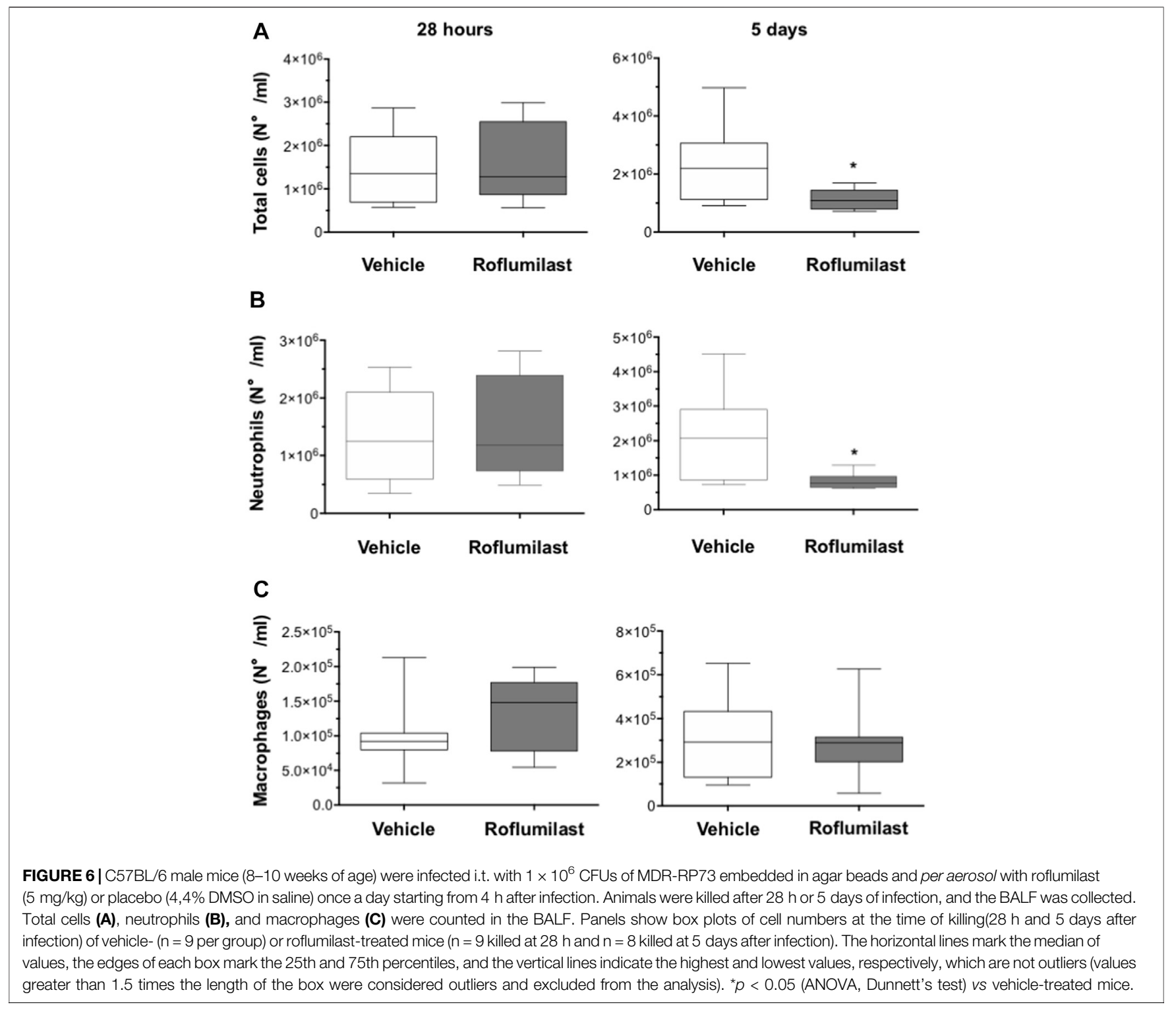

bacterial load in the BALF and lungs at $28 \mathrm{~h}$ and 5 days was not affected by roflumilast treatment and decreased by approximately an order of magnitude 5 days after infection compared to the 28-h time point, in all groups of treatment (Supplementary Figure S1). Likewise, the amounts of KC, TNF- $\alpha$, and MIP2 decreased at 5 days and were not significantly affected by roflumilast (Supplementary Figure S2).

Correlation analyses between inflammation markers and the body weight loss at 5 days after infection, including all animals treated with roflumilast or a vehicle of both experimental protocols (per os or intratracheal) (Supplementary Figure S3), showed that the number of neutrophils (A) as well as the amount of free DNA (B), KC (D), and total proteins (F) in the BALF, positively correlated with the weight loss. In contrast, no or very weak correlations were found between the number of macrophages (e), the amount of IL-1 $\beta$ (G), TNF- $\alpha(C)$, and weight loss. Unexpectedly, the bacterial load (the $\mathrm{CFU}$ in homogenates of lung tissue) did not correlate with the weight loss $(\mathrm{H})$.

\section{DISCUSSION}

The mechanisms of neutrophilic inflammation in CF lung disease remain to be fully understood and no established pharmacological treatments are currently available to control this process. However, recent studies strongly suggest that NETs are main determinants of lung inflammation and damage in CF. Therefore, identifying therapies that preserve the positive effects of neutrophils, while reducing the detrimental effects of NETs and cytotoxic components, is essential for achieving innovative therapeutic advances (Khan et al., 2019). Here, we provide evidence that PDE4 inhibition prevents NETosis in, in vitro and in vivo, CF-relevant models. 


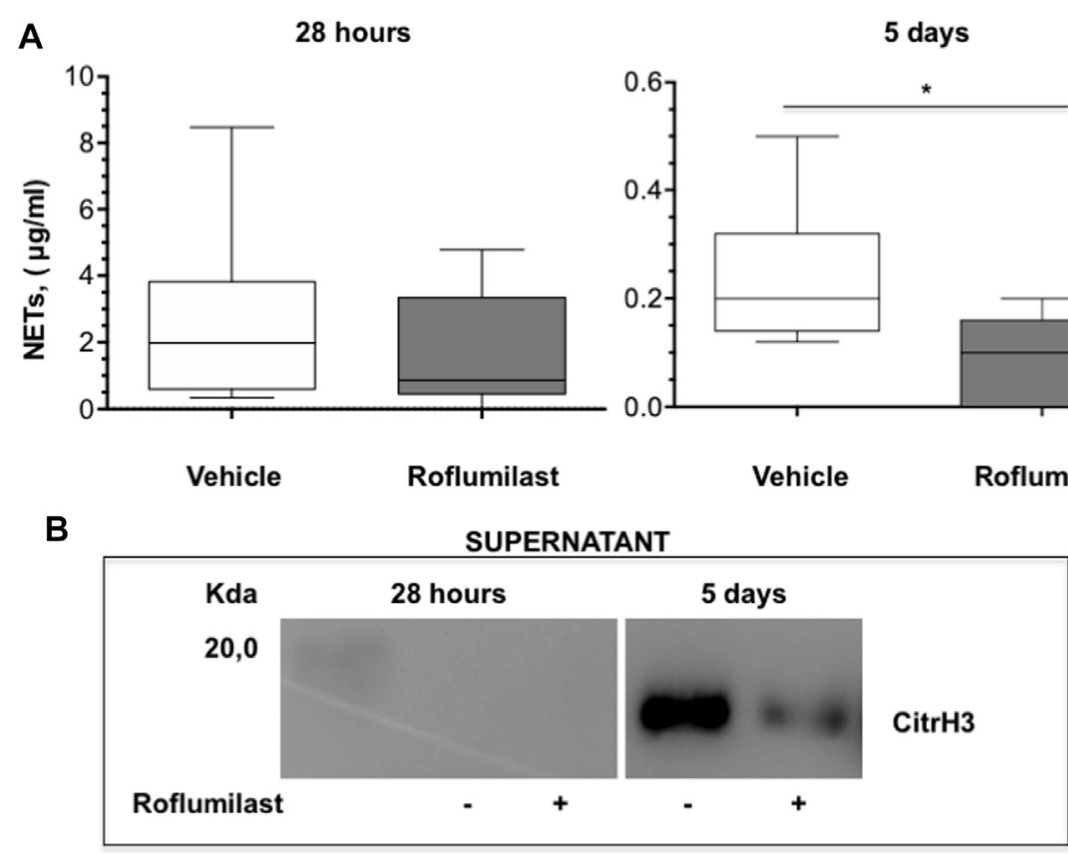

C CELL LYSATE

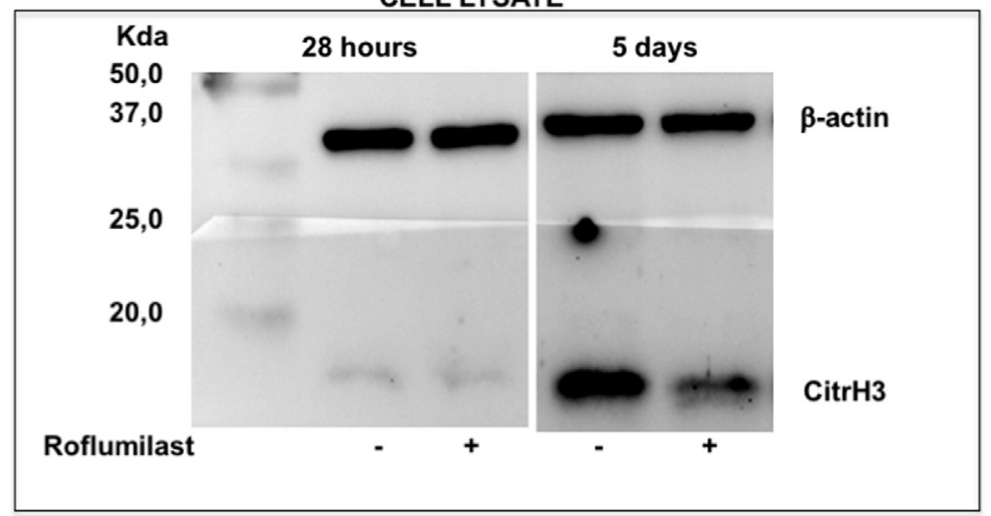

FIGURE 7 | C57BL/6 male mice were treated as described in Figure 6 and killed after 28 h or 5 days of infection. The BALF was collected for measurement of free DNA in supernatants and citrullinated histone $\mathrm{H} 3$ in supernatants and in cell lysates. (A) shows box plots of free-DNA values at the time of killing (28 h and 5 days after infection) of vehicle- ( $\mathrm{n}=9$ per group) or roflumilast-treated mice ( $\mathrm{n}=9$ killed at $28 \mathrm{~h}$ and $\mathrm{n}=8$ killed at 5 days after infection). ${ }^{*} p<0.05$ (ANOVA, Dunnett's test) vs vehicletreated mice. The presence of citrullinated histone $\mathrm{H} 3$ was analyzed by Western blotting in BALF supernatants (B) as well as in lysates of BALF cells from mice killed at $28 \mathrm{~h}$ and 5 days after infection (C). Pools of BALF supernatants and of cell lysates from all animals of each group were subjected to Western blotting using a monoclonal antibody which recognizes mouse with citrullinated histone $\mathrm{H} 3$.

In this work, we tested the selective PDE4 inhibitor roflumilast mainly because this drug has been tested in phase II and III clinical trials (Rabe, 2011) and has been approved as an adjuvant to reduce the risk of exacerbation in patients with severe COPD (Schudt et al., 2011). Like CF, COPD is, in fact, characterized by excessive neutrophilic lung infiltration as well as by an imbalance in the oxidant/antioxidant and protease/antiprotease equilibrium, two neutrophil-mediated processes, regarded as major determinants of the progressive lung damage in both diseases. PDE4 blockade in lung immune cells prevents the progression of inflammation in COPD (Rabe, 2011; Baye, 2012). Moreover, roflumilast reduced neutrophil and eosinophil accumulation in BAL of healthy volunteers subjected to segmental administration of endotoxin (Hohlfeld et al., 2008). Further indication for the clinical use of PDE4 inhibitors in CF derives from the evidence that PDE4 inhibition rescues CFTR activity in varying experimental settings (Blanchard et al., 2014).

RNO reduced NET release by neutrophils in vitro (Figures 1-3). This was clearly demonstrated by combining confocal microscopy with measurements of free DNA and citrullinated histone H3. These analyses were conducted on neutrophils from healthy donors, exposed or not exposed to CFTRinh-172 to mimic a CF status, and on neutrophils from donors with $\mathrm{CF}$ 

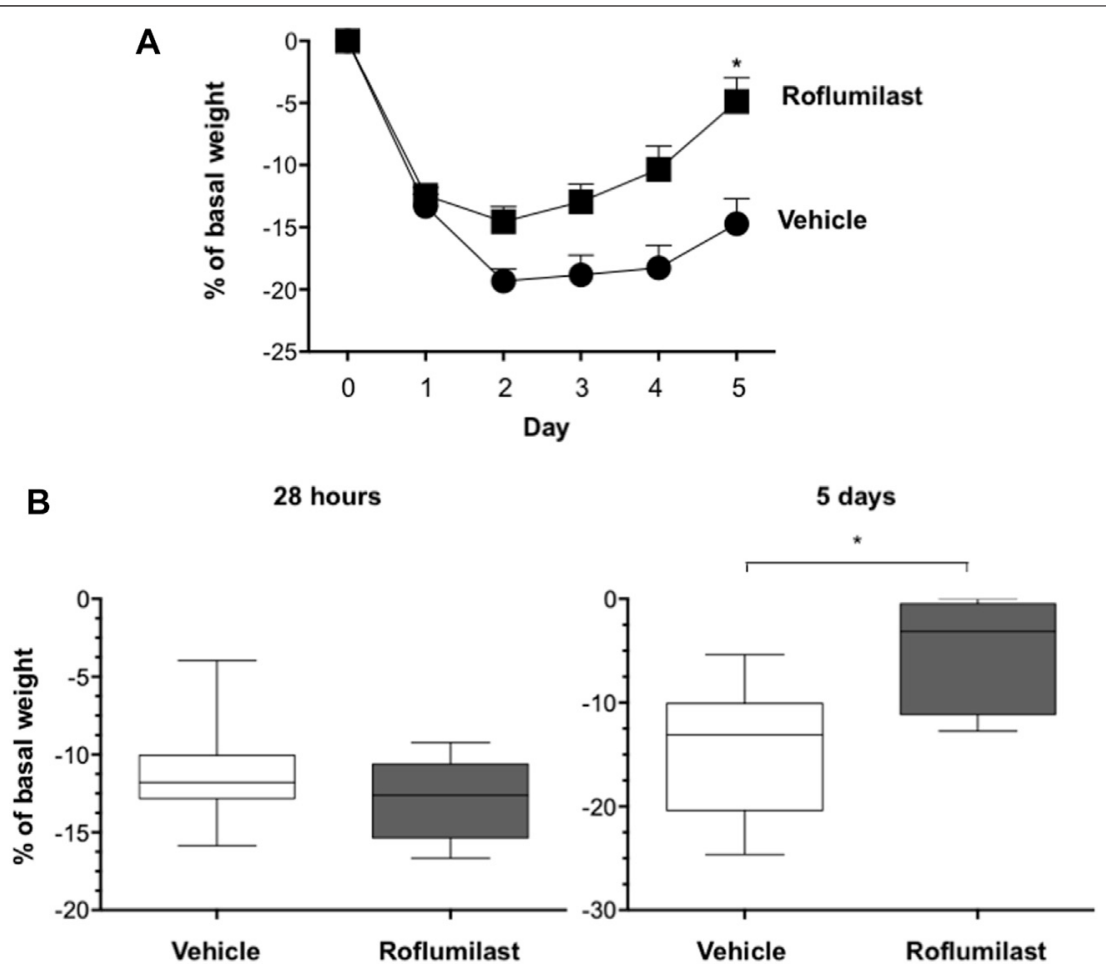

FIGURE 8|C57BL/6 male mice were treated as described in Figure 6. (A). Body weight of mice was monitored daily before treatment to evaluate the health status. ${ }^{*} p<0.05$ (ANOVA, Dunnett test) vs vehicle-treated mice. (B) shows box plots of weight loss values at the time of killing(28 $\mathrm{h}$ and 5 days after infection) of vehicle- or roflumilast-treated mice. ${ }^{*} p<0.05$ (Student's t-test) vs vehicle-treated mice.

with different CFTR mutations and disease severity (Table1). The overall picture emerging from these experiments shows that while CFTR blockade did not modify the entity of NET release, RNO was significantly more potent at inhibiting NETosis in CFTRinh172-treated normal neutrophils as well as in CF neutrophils, compared to untreated normal neutrophils. While confirming previous data from our and other's laboratory (Shishikura et al., 2016; Totani et al., 2016), the present results provide the novel observation that PDE4 blockade is more efficient at reducing NETs under CF conditions, suggesting that impairment of CFTR function induces signaling events that favor the activity of the PDE4 inhibitor.

In addition to reducing NETs, RNO preserved neutrophil integrity as well as the stability of MPO-containing intracellular granules (Figure 4). These are key events in neutrophil-driven inflammation since the release of the content of proteolytic enzymes in the airways, stored in neutrophil granules, sustains CF inflammation and lung damage. Along these lines, the observation that RNO preserves both neutrophil integrity and the apoptotic process further supports the hypothesis that PDE4 inhibition may sustain the resolution program of inflammation, which requires a discrete neutrophil apoptosis to be preserved. A recent work links NETosis with a delayed neutrophil apoptosis in CF and suggests that promotion of apoptosis may allow more appropriate neutrophil disposal, decreasing NET formation and inflammation (Gray et al., 2018). The existence of a functional link between the two processes, NETosis and apoptosis, is also supported by the results of Remijsen et al. (2011) showing that inhibition of either autophagy or NADPH oxidase activity, which are essential for NET formation, results in cell death characterized by hallmarks of apoptosis, suggesting that switching-on an apoptosis program might function as a stop signal for NETosis and vice versa. In keeping with this, our results in in vitro models demonstrate that PDE4 blockade reduces NETosis while preserving apoptosis, and this is particularly relevant in CF neutrophils. Biochemical pathways mediated by cAMP-activated PKA and regulating kinases belonging to the src family as well as the PI3K/Akt pathway appear to play a relevant role in this effect (Sousa et al., 2010; Totani et al., 2014, 2016).

In vitro data were confirmed in a preclinical mouse model of respiratory infection by Pseudomonas aeruginosa, which often colonizes the airways of patients with CF. We used two different routes of roflumilast administration, by gavage or intratracheal, and monitored markers of neutrophilic inflammation at short $(28 \mathrm{~h})$ and extended time (5 days). The results showed that while these markers were barely affected by the drug given per os, the aerosol administration was effective in reducing the accumulation of neutrophils at 5 days but not after $28 \mathrm{~h}$ of infection. Accordingly, roflumilast-treated mice showed a significant reduction in the accumulation of free DNA in the BALF at 5 days, as well as reduced citrullination of histone $\mathrm{H} 3$, both in BALF supernatants and cells, thus conclusively demonstrating that PDE4 inhibition controls key biochemical steps necessary for 
NET formation in vivo. Of note, roflumilast did not modify DNA release after $28 \mathrm{~h}$ of infection, and at this early time point, we did not observe measurable amounts of citrullinated histone $\mathrm{H} 3$. Although we are unable to provide a clear explanation for this finding, we may hypothesize that, at this early time point, 1) DNA release is not a result of NETosis and 2) PAD4-independent mechanisms may be responsible for NETosis. Further studies are necessary to clarify this point.

In this model, roflumilast effects on neutrophil functions correlated with a more rapid weight recovery, which reached the statistical significance at 5 days of infection (Figure 8A). The hypothesis that roflumilast improves the animal well-being by modulating neutrophil recruitment and function was supported by the observation of a direct correlation between the neutrophil number and free DNA with body weight (Supplementary Figure S3). On the contrary, the bacterial load, which was not appreciably modified by roflumilast, was not an influent.

Consistent with this scenario, free DNA accumulated in the BALF of Pseudomonas aeruginosa-infected mice positively correlated with neutrophil counts. In this condition, free DNA may be largely represented by NETs, as indicated by histone $\mathrm{H} 3$ data. Thus, NET release appears to represent a neutrophil function mainly involved in the pathogenesis of lung inflammation during Pseudomonas aeruginosa infection. This interpretation is consistent with recent evidence that free DNA, abundant in CF sputum, shows NET characteristics (Dwyer et al., 2014). Moreover, in the airways of people with CF, NET components, such as elastase and other granule proteins, perpetuate lung damage and inflammation (Marcos et al., 2015; Dittrich et al., 2018), while decondensed chromatin, the main structure of NETs, increases the viscosity of endobronchial secretions, further hampering mucociliary clearance. Clinical observations also confirm the pathogenetic role of NETs in CF, by revealing a positive correlation between the impairment of respiratory function and the level of free DNA or elastase in CF airways, or the level of MPO and antibodies to PAD4 in circulating blood (Marcos et al., 2015; Dittrich et al., 2018; Yadav et al., 2019).

In summary, our study describes a series of pharmacological activities associated with PDE4 blockade in neutrophils, which could be beneficial in a CF clinical setting. Our data, showing that PDE4 inhibitors may sustain a local signal driving neutrophilic inflammation toward physiological resolution, indicate that PDE4 may be a potential novel target to promote a "correction" of neutrophilic inflammation, rather than a complete suppression, which could be detrimental. As PDE4 inhibitors have been recently approved for clinical use in COPD (Rabe, 2011; Wedzicha et al., 2016) and psoriasis (Rich et al., 2016), our present results encourage further research to validate the use of these drugs in patients with $\mathrm{CF}$.

\section{DATA AVAILABILITY STATEMENT}

The original contributions presented in the study are included in the article/Supplementary Material. Further inquiries can be directed to the corresponding authors.

\section{ETHICS STATEMENT}

The studies involving human participants were reviewed and approved by Comitato Etico Province di L'Aquila e Teramo. The patients/participants provided their written informed consent to participate in this study. The animal study was reviewed and approved by the Italian Ministry of Health (protocol no. 549 and 733).

\section{AUTHOR CONTRIBUTIONS}

LT and VE designed the experiments, analyzed the data, supervised the study, and wrote the manuscript; CA, AP, RPe, and AS performed in vitro studies on NET release, DNA measurement, and confocal microscopy; GDE performed biochemical studies and Western blot analysis; NM performed flow cytometry; RPl performed cytokine measurements; AR, SR, $\mathrm{IF}$, and $\mathrm{AB}$ conducted in vivo experiments; $\mathrm{PM}$ recruited patients; $\mathrm{MR}$ and $\mathrm{AB}$ wrote the manuscript.

\section{FUNDING}

This work was supported by the Italian Cystic Fibrosis Research Foundation (project FFC\#21/2011, sponsor Philip Watch-Morellato and Sector Group and FFC\#16/2013 sponsor: LIFC con Associazioni Regionali per la Campagna Nazionale FFC, LIFC Associazione Emiliana Onlus, Delegazione FFC di Lecce) to VE; by Fondazione Negri Sud Onlus (VE); MIUR D.M. 44/08 (VE); and by the Italian Ministry of Health (L548/93 to V.E. and M.R.). RPl, AP, and RPe received fellowships by the Italian Cystic Fibrosis Research Foundation. $\mathrm{AR}, \mathrm{SR}, \mathrm{IDF}$, and $\mathrm{AB}$ are supported by the Italian Cystic Fibrosis Research Foundation (CFaCore).

\section{ACKNOWLEDGMENTS}

We are indebted with all people with CF who volunteered for this study. We also thank Hermann Tenor (TOPADUR Pharma AG, Schlieren, Switzerland) for the fruitful discussion. This study was initiated at the Fondazione Mario Negri Sud where most of the in vitro experiments were performed until the closure of the Institution, because of failure, in May 2015. Thereafter, the project was continued and concluded at the Department of Medical, Oral and Biotechnological Sciences, G. D'Annunzio University of Chieti-Pescara; Center for Advanced Studies and Technology (CAST), G. D’Annunzio University of ChietiPescara.

\section{SUPPLEMENTARY MATERIAL}

The Supplementary Material for this article can be found online at: https://www.frontiersin.org/articles/10.3389/fphar.2021.702677/ full\#supplementary-material 


\section{REFERENCES}

Ariga, M., Neitzert, B., Nakae, S., Mottin, G., Bertrand, C., Pruniaux, M. P., et al. (2004). Nonredundant Function of Phosphodiesterases $4 \mathrm{D}$ and $4 \mathrm{~B}$ in Neutrophil Recruitment to the Site of Inflammation. J. Immunol. 173, 7531-7538. doi:10.4049/jimmunol.173.12.7531

Baye, J. (2012). Roflumilast (Daliresp): a Novel Phosphodiesterase-4 Inhibitor for the Treatment of Severe Chronic Obstructive Pulmonary Disease. $P$ T 37, 149-161.

Blanchard, E., Zlock, L., Lao, A., Mika, D., Namkung, W., Xie, M., et al. (2014). Anchored PDE4 Regulates Chloride Conductance in Wild-type and $\triangle \mathrm{F} 508$ CFTR Human Airway Epithelia. FASEB j. 28, 791-801. doi:10.1096/fj.13240861

Bragonzi, A. (2010). Murine Models of Acute and Chronic Lung Infection with Cystic Fibrosis Pathogens. Int. J. Med. Microbiol. 300, 584-593. doi:10.1016/ j.ijmm.2010.08.012

Brinkmann, V., Reichard, U., Goosmann, C., Fauler, B., Uhlemann, Y., Weiss, D. S., et al. (2004). Neutrophil Extracellular Traps Kill Bacteria. Science 303, 1532-1535. doi:10.1126/science.1092385

Bundschuh, D. S., Eltze, M., Barsig, J., Wollin, L., Hatzelmann, A., and Beume, R. (2001). In Vivo Efficacy in Airway Disease Models of Roflumilast, a Novel Orally Active PDE4 Inhibitor. J. Pharmacol. Exp. Ther. 297, 280-290.

Cheng, O. Z., and Palaniyar, N. (2013). NET Balancing: a Problem in Inflammatory Lung Diseases. Front. Immunol. 4, 1. doi:10.3389/fimmu.2013.00001

Cutone, A., Lepanto, M. S., Rosa, L., Scotti, M. J., Rossi, A., Ranucci, S., et al. (2019). Aerosolized Bovine Lactoferrin Counteracts Infection, Inflammation and Iron Dysbalance in A Cystic Fibrosis Mouse Model of Pseudomonas aeruginosa Chronic Lung Infection. Int. J. Mol. Sci. 20, 2028. doi:10.3390/ijms20092128

Davis, S. D., and Ferkol, T. (2013). Identifying the Origins of Cystic Fibrosis Lung Disease. N. Engl. J. Med. 368, 2026-2028. doi:10.1056/nejme1303487

Dittrich, A. S., Kühbandner, I., Gehrig, S., Rickert-Zacharias, V., Twigg, M., Wege, S., et al. (2018). Elastase Activity on Sputum Neutrophils Correlates with Severity of Lung Disease in Cystic Fibrosis. Eur. Respir. J. 51, 1701910. doi:10.1183/13993003.01910-2017

Dwyer, M., Shan, Q., D’Ortona, S., Maurer, R., Mitchell, R., Olesen, H., et al. (2014). Cystic Fibrosis Sputum DNA Has NETosis Characteristics and Neutrophil Extracellular Trap Release Is Regulated by Macrophage Migration-Inhibitory Factor. J. Innate Immun. 6, 765-779. doi:10.1159/000363242

Evangelista, V., Pamuklar, Z., Piccoli, A., Manarini, S., Dell'Elba, G., Pecce, R., et al. (2007). Src Family Kinases Mediate Neutrophil Adhesion to Adherent Platelets. Blood 109, 2461-2469. doi:10.1182/blood-2006-06-029082

Facchini, M., De Fino, I., Riva, C., and Bragonzi, A. (2014). Long Term Chronic Pseudomonas aeruginosa Airway Infection in Mice. J. Vis. Exp. 17, 51019. doi:10.3791/51019

Fuchs, T. A., Brill, A., Duerschmied, D., Schatzberg, D., Monestier, M., Myers, D. D., et al. (2010). Extracellular DNA Traps Promote Thrombosis. Proc. Natl. Acad. Sci. 107, 15880-15885. doi:10.1073/pnas.1005743107

Gray, R. D., Hardisty, G., Regan, K. H., Smith, M., Robb, C. T., Duffin, R., et al. (2018). Delayed Neutrophil Apoptosis Enhances NET Formation in Cystic Fibrosis. Thorax 73, 134-144. doi:10.1136/thoraxjnl-2017-210134

Hartl, D., Latzin, P., Hordijk, P., Marcos, V., Rudolph, C., Woischnik, M., et al. (2007). Cleavage of CXCR1 on Neutrophils Disables Bacterial Killing in Cystic Fibrosis Lung Disease. Nat. Med. 13, 1423-1430. doi:10.1038/nm1690

Hohlfeld, J. M., Schoenfeld, K., Lavae-Mokhtari, M., Schaumann, F., Mueller, M., Bredenbroeker, D., et al. (2008). Roflumilast Attenuates Pulmonary Inflammation upon Segmental Endotoxin challenge in Healthy Subjects: a Randomized Placebo-Controlled Trial. Pulm. Pharmacol. Ther. 21, 616-623. doi:10.1016/j.pupt.2008.02.002

Jin, S.-L. C., Lan, L., Zoudilova, M., and Conti, M. (2005). Specific Role of Phosphodiesterase $4 \mathrm{~B}$ in Lipopolysaccharide-Induced Signaling in Mouse Macrophages. J. Immunol. 175, 1523-1531. doi:10.4049/jimmunol.175.3.1523

Jin, S. L. C., and Conti, M. (2002). Induction of the Cyclic Nucleotide Phosphodiesterase PDE4B Is Essential for LPS-Activated TNF- Responses. Proc. Natl. Acad. Sci. 99, 7628-7633. doi:10.1073/pnas.122041599

Kessenbrock, K., Krumbholz, M., Schönermarck, U., Back, W., Gross, W. L., Werb, Z., et al. (2009). Netting Neutrophils in Autoimmune Small-Vessel Vasculitis. Nat. Med. 15, 623-625. doi:10.1038/nm.1959
Khan, M. A., Ali, Z. S., Sweezey, N., Grasemann, H., and Palaniyar, N. (2019). Progression of Cystic Fibrosis Lung Disease from Childhood to Adulthood: Neutrophils, Neutrophil Extracellular Trap (NET) Formation, and NET Degradation. Genes 10, 183. doi:10.3390/genes10030183

Li, M., Lin, C., Deng, H., Strnad, J., Bernabei, L., Vogl, D. T., et al. (2020). A Novel Peptidylarginine Deiminase 4 (PAD4) Inhibitor BMS-P5 Blocks Formation of Neutrophil Extracellular Traps and Delays Progression of Multiple Myeloma. Mol. Cancer Ther. 19, 1530-1538. doi:10.1158/1535-7163.mct-19-1020

Marcos, V., Zhou-Suckow, Z., Önder Yildirim, A., Bohla, A., Hector, A., Vitkov, L., et al. (2015). Free DNA in Cystic Fibrosis Airway Fluids Correlates with Airflow Obstruction. Mediators Inflamm. 2015, 1-11. doi:10.1155/2015/408935

McKeon, D. J., Condliffe, A. M., Cowburn, A. S., Cadwallader, K. C., Farahi, N., Bilton, D., et al. (2008). Prolonged Survival of Neutrophils from Patients with F508 CFTR Mutations. Thorax 63, 660-661. doi:10.1136/thx.2008.096834

Mondal, S., and Thompson, P. R. (2019). Protein Arginine Deiminases (PADs): Biochemistry and Chemical Biology of Protein Citrullination. Acc. Chem. Res. 52, 818-832. doi:10.1021/acs.accounts.9b00024

Moriceau, S., Lenoir, G., and Witko-Sarsat, V. (2010). In Cystic Fibrosis Homozygotes and Heterozygotes, Neutrophil Apoptosis Is Delayed and Modulated by Diamide or Roscovitine: Evidence for an Innate Neutrophil Disturbance. J. Innate Immun. 2, 260-266. doi:10.1159/000295791

Neeli, I., Khan, S. N., and Radic, M. (2008). Histone Deimination as a Response to Inflammatory Stimuli in Neutrophils. J. Immunol. 180, 1895-1902. doi:10.4049/ jimmunol.180.3.1895

Papayannopoulos, V., Metzler, K. D., Hakkim, A., and Zychlinsky, A. (2010). Neutrophil Elastase and Myeloperoxidase Regulate the Formation of Neutrophil Extracellular Traps. J. Cel Biol. 191, 677-691. doi:10.1083/ jcb.201006052

Paroni, M., Moalli, F., Nebuloni, M., Pasqualini, F., Bonfield, T., Nonis, A., et al. (2013). Response of CFTR-Deficient Mice to Long-Term Chronic Pseudomonas aeruginosa Infection and PTX3 Therapy. J. Infect. Dis. 208, 130-138. doi:10.1093/infdis/jis636

Rabe, K. F. (2011). Update on Roflumilast, a Phosphodiesterase 4 Inhibitor for the Treatment of Chronic Obstructive Pulmonary Disease. Br. J. Pharmacol. 163, 53-67. doi:10.1111/j.1476-5381.2011.01218.x

Remijsen, Q., Berghe, T. V., Wirawan, E., Asselbergh, B., Parthoens, E., De Rycke, R., et al. (2011). Neutrophil Extracellular Trap Cell Death Requires Both Autophagy and Superoxide Generation. Cell Res. 21, 290-304. doi:10.1038/ cr.2010.150

Rich, P., Gooderham, M., Bachelez, H., Goncalves, J., Day, R. M., Chen, R., et al. (2016). Apremilast, an Oral Phosphodiesterase 4 Inhibitor, in Patients with Difficult-To-Treat Nail and Scalp Psoriasis: Results of 2 Phase III Randomized, Controlled Trials (ESTEEM 1 and ESTEEM 2). J. Am. Acad. Dermatol. 74, 134-142. doi:10.1016/j.jaad.2015.09.001

Sanz, M.-J., Alvarez, A., Piqueras, L., Cerdá, M., Issekutz, A. C., Lobb, R. R., et al. (2002). Rolipram Inhibits Leukocyte-Endothelial Cell Interactions In Vivo through P- and E-Selectin Downregulation. Br. J. Pharmacol. 135, 1872-1881. doi:10.1038/sj.bjp.0704644

Sanz, M.-J., Cortijo, J., Taha, M. A., Cerdá-Nicolás, M., Schatton, E., Burgbacher, B., et al. (2007). Roflumilast Inhibits Leukocyte-Endothelial Cell Interactions, Expression of Adhesion Molecules and Microvascular Permeability. $\mathrm{Br}$. J. Pharmacol. 152, 481-492. doi:10.1038/sj.bjp.0707428

Sanz, M. J., Cortijo, J., and Morcillo, E. J. (2005). PDE4 Inhibitors as New Antiinflammatory Drugs: Effects on Cell Trafficking and Cell Adhesion Molecules Expression. Pharmacol. Ther. 106, 269-297. doi:10.1016/ j.pharmthera.2004.12.001

Schudt, C., Hatzelmann, A., Beume, R., and Tenor, H. (2011). Phosphodiesterase Inhibitors: History of Pharmacology. Handb Exp. Pharmacol. 204, 1-46. doi:10.1007/978-3-642-17969-3_1

Shishikura, K., Horiuchi, T., Sakata, N., Trinh, D.-A., Shirakawa, R., Kimura, T., et al. (2016). Prostaglandin E2inhibits Neutrophil Extracellular Trap Formation through Production of Cyclic AMP. Br. J. Pharmacol. 173, 319-331. doi:10.1111/bph.13373

Sly, P. D., Gangell, C. L., Chen, L., Ware, R. S., Ranganathan, S., Mott, L. S., et al. (2013). Risk Factors for Bronchiectasis in Children with Cystic Fibrosis. N. Engl. J. Med. 368, 1963-1970. doi:10.1056/nejmoal301725

Sousa, L. P., Lopes, F., Silva, D. M., Tavares, L. P., Vieira, A. T., Rezende, B. M., et al. (2010). PDE4 Inhibition Drives Resolution of Neutrophilic Inflammation by 
Inducing Apoptosis in a PKA-PI3K/Akt-dependent and NF-kb-independent Manner. J. Leukoc. Biol. 87, 895-904. doi:10.1189/jlb.0809540

Thiam, H. R., Wong, S. L., Qiu, R., Kittisopikul, M., Vahabikashi, A., Goldman, A. E., et al. (2020). NETosis Proceeds by Cytoskeleton and Endomembrane Disassembly and PAD4-Mediated Chromatin Decondensation and Nuclear Envelope Rupture. Proc. Natl. Acad. Sci. USA. 117, 7326-7337. doi:10.1073/ pnas. 1909546117

Tirouvanziam, R. (2006). Neutrophilic Inflammation as a Major Determinant in the Progression of Cystic Fibrosis. Drug News Perspect. 19, 609-614. doi:10.1358/dnp.2006.19.10.1068008

Totani, L., Amore, C., Di Santo, A., Dell'Elba, G., Piccoli, A., Martelli, N., et al. (2016). Roflumilast Inhibits Leukocyte-Platelet Interactions and Prevents the Prothrombotic Functions of Polymorphonuclear Leukocytes and Monocytes. J. Thromb. Haemost. 14, 191-204. doi:10.1111/jth.13173

Totani, L., Piccoli, A., Dell'Elba, G., Concetta, A., Di Santo, A., Martelli, N., et al. (2014). Phosphodiesterase Type 4 Blockade Prevents Platelet-Mediated Neutrophil Recruitment at the Site of Vascular Injury. Arterioscler Thromb. Vasc. Biol. 34, 1689-1696. doi:10.1161/atvbaha.114.303939

Wang, Y., Li, M., Stadler, S., Correll, S., Li, P., Wang, D., et al. (2009). Histone Hypercitrullination Mediates Chromatin Decondensation and Neutrophil Extracellular Trap Formation. J. Cel Biol. 184, 205-213. doi:10.1083/ jcb.200806072

Wedzicha, J., Calverley, P., and Rabe, K. (2016). Roflumilast: a Review of its Use in the Treatment of COPD. Copd 11, 81-90. doi:10.2147/copd.s89849

Yadav, R., Yoo, D.-G., Kahlenberg, J. M., Bridges, S. L., Oni, O., Huang, H., et al. (2019). Systemic Levels of Anti-PAD4 Autoantibodies Correlate with Airway
Obstruction in Cystic Fibrosis. J. Cystic Fibrosis. 18, 636-645. doi:10.1016/ j.jcf.2018.12.010

Young, R. L., Malcolm, K. C., Kret, J. E., Caceres, S. M., Poch, K. R., Nichols, D. P., et al. (2011). Neutrophil Extracellular Trap (NET)-Mediated Killing of Pseudomonas aeruginosa: Evidence of Acquired Resistance within the CF Airway, Independent of CFTR. PLoS One. 6, e23637. doi:10.1371/ journal.pone.0023637

Conflict of Interest: The authors declare that the research was conducted in the absence of any commercial or financial relationships that could be construed as a potential conflict of interest.

Publisher's Note: All claims expressed in this article are solely those of the authors and do not necessarily represent those of their affiliated organizations, or those of the publisher, the editors, and the reviewers. Any product that may be evaluated in this article, or claim that may be made by its manufacturer, is not guaranteed or endorsed by the publisher.

Copyright (c) 2021 Totani, Amore, Piccoli, Dell'Elba, Di Santo, Plebani, Pecce, Martelli, Rossi, Ranucci, De Fino, Moretti, Bragonzi, Romano and Evangelista. This is an open-access article distributed under the terms of the Creative Commons Attribution License (CC BY). The use, distribution or reproduction in other forums is permitted, provided the original author(s) and the copyright owner(s) are credited and that the original publication in this journal is cited, in accordance with accepted academic practice. No use, distribution or reproduction is permitted which does not comply with these terms. 